\title{
THE EXTENSION OF NORMS ON SUBGROUPS OF FREE TOPOLOGICAL GROUPS
}

\author{
SIDNEY A. MORRIS AND PETER NICKOLAS
}

\begin{abstract}
A norm on a group $\boldsymbol{G}$ is a nonnegative real-valued function $\boldsymbol{N}$ which is zero at the identity and satisfies $N\left(x y^{-1}\right)<N(x)+N(y)$, for $x, y \in G$. Let $F(X)$ be the free topological group on a space $X$. Bicknell and Morris have shown that any norm on a subgroup of $F(X)$ generated by a finite subset of $X$ may be extended to a continuous norm on the whole of $F(X)$. In this note a very direct and simple proof of this theorem is given.
\end{abstract}

Our object in this note is to provide a very simple and direct proof of a theorem of Bicknell and Morris [1] relating to norms on free topological groups. Recall that a norm on a topological group $G$ is a function $N: G \rightarrow \mathbf{R}$ which is zero at the identity of $G$ and for which $N\left(x y^{-1}\right) \leqslant N(x)+N(y)$ for each $x, y \in G$; such an $N$ is clearly nonnegative. The theorem we refer to states that if $F(X)$ is the (Markov) free topological group on a completely regular Hausdorff space $X$, and if $H$ is the subgroup of $F(X)$ generated by any finite subset of $X$, then any norm on $H$ can be extended to a continuous norm on $F(X)$.

The main tool used by Bicknell and Morris in their proof is a construction of Brown and Morris [2] (cf. [3]) which in particular embeds any topological group $G$ in a contractible, locally contractible topological group $G^{*}$. The groups $G^{*}$ play a central role in our proof also, but we are able to make a significant simplification by employing a topology on $G^{*}$ which is (in general) different from that of Brown and Morris.

We now discuss in detail the ideas we shall need later.

For any space $X$ we let $X^{*}$ be the set of continuous-from-the-right step functions from the half-open unit interval $[0,1)$ to $X$; that is, functions $f$ for which there is a partition $0=a_{0}<a_{1}<\cdots<a_{n-1}<a_{n}=1$ of $[0,1]$ such that $f$ is constant on each $\left[a_{i-1}, a_{i}\right)$. There is an injection $i: X \rightarrow X^{*}$ defined by $i(x)(t)=x, t \in[0,1)$, $x \in X$. It is easy to see that if $X$ is a universal algebra of a given type (for example, a group) then $X^{*}$ is a universal algebra of the same type if the operations are defined pointwise. Furthermore, $i$ is a morphism.

Recall that a pseudometric $\nu$ on a topological group $G$ is said to be left-invariant if $\nu(a x, a y)=\nu(x, y)$ for all $a, x, y \in G$. There is a natural one-to-one correspondence between left-invariant pseudometrics and norms on $G$. Specifically, the formula $\nu(x, y)=N\left(y^{-1} x\right), x, y \in G$, defines a left-invariant pseudometric if $N$ is a norm, while the formula $N(x)=\nu(x, e), x \in G$, defines a norm if $\nu$ is a

Received by the editors September 4, 1979.

1980 Mathematics Subject Classification. Primary 22A05.

Key words and phrases. Norm on a topological group, free topological group. 
left-invariant pseudometric and $e$ is the identity of $G$; it is clear that these are inverse constructions. Any pair $\nu, N$ as just described have natural extensions $\nu^{*}$, $N^{*}$ on $G^{*}$ defined by

$$
\nu^{*}(f, g)=\int_{0}^{1} \nu(f(t), g(t)) d t \quad \text { and } N^{*}(f)=\int_{0}^{1} N(f(t)) d t,
$$

for $f, g \in G^{*}$. We observe that $\nu^{*}$ is left-invariant, and that it is a metric if $\nu$ is. It is straightforward to check that these extension processes commute with the one-toone correspondence mentioned above. Throughout this paper we shall use the Roman letters $N$ and $L$ to represent norms, and the corresponding Greek letters $\nu$ and $\lambda$ for the associated pseudometrics; further, if any of these letters is assigned an asterisk (as in $N^{*}, L^{*}, \nu^{*}$ or $\lambda^{*}$ ), the resulting symbol will be taken without explanation to refer to the appropriate extended norm or pseudometric.

Suppose that $G$ is a topological group whose topology is induced by a left-invariant pseudometric $\nu$. (Note that a left-invariant pseudometric on a group does not always make the group operations continuous.) Proposition 5 of [2] and the example give in [1] show that the topology $\tau(\nu)$ induced on $G^{*}$ by $\nu^{*}$ coincides with the usual Brown-Morris topology if and only if $\nu$ is bounded; the nature of $\tau(\nu)$ when $\nu$ is unbounded has not previously been examined. As a consequence, the work of [1] is complicated by the need to ensure that all pseudometrics used are bounded. We are able to do away with this restriction, and greatly simplify the proof of the norm extension theorem, by means of the result below, which assures us that the topology $\tau(\nu)$ has the properties we require, whether or not $\nu$ is bounded.

THEOREM 1. If $G$ is a topological group with topology induced by a left-invariant pseudometric $\nu$, then $G^{*}$, with the topology $\tau(\nu)$ induced by $\nu^{*}$, is a topological group, and is path-connected.

Proof. To prove that $G^{*}$ is a topological group, we shall show directly that the function $(f, g) \mapsto f g^{-1}, f, g \in G^{*}$, is continuous on $G^{*} \times G^{*}$.

Let $e$ be the identity of $G$. By the left-invariance of $\nu$ we have, for $a, b, a_{0}, b_{0} \in$ G,

$$
\begin{aligned}
\nu\left(a b^{-1}, a_{0} b_{0}^{-1}\right) & =\nu\left(a_{0}^{-1} a b^{-1}, b_{0}^{-1}\right) \\
& \leqslant \nu\left(a_{0}^{-1} a b^{-1}, e\right)+\nu\left(e, b_{0}^{-1}\right)=\nu\left(b^{-1}, a^{-1} a_{0}\right)+\nu\left(b_{0}, e\right) \\
& \leqslant \nu\left(b^{-1}, e\right)+\nu\left(e, a^{-1} a_{0}\right)+\nu\left(b_{0}, e\right)
\end{aligned}
$$

so that

$$
\nu\left(a b^{-1}, a_{0} b_{0}^{-1}\right) \leqslant \nu\left(a, a_{0}\right)+\nu\left(b, b_{0}\right)+2 \nu\left(b_{0}, e\right) .
$$

Now fix $\left(f_{0}, g_{0}\right) \in G^{*} \times G^{*}$ and set $M=\max \left\{\nu\left(g_{0}(t), e\right): t \in[0,1)\right\}$. Given $\varepsilon>$ 0 , the facts that $f_{0}$ and $g_{0}$ take on only finitely many values, and that the group operations in $G$ are continuous, show that there exists a $\delta_{1}>0$ such that $\nu\left(a b^{-1}, f_{0} g_{0}^{-1}(t)\right)<\varepsilon / 3$ whenever $t$ is such that both $\nu\left(a, f_{0}(t)\right)$ and $\nu\left(b, g_{0}(t)\right)$ are less than $\delta_{1}$. We may assume that $\delta_{1}<\min (1, \varepsilon / 12(M+1))$. Then if $\delta=$ $\min \left(\delta_{1}^{2}, \varepsilon / 6\right)$ and both $\nu^{*}\left(f, f_{0}\right)$ and $\nu^{*}\left(g, g_{0}\right)$ are less than $\delta$, we see that 
$\nu\left(f(t), f_{0}(t)\right)$ and $\nu\left(g(t), g_{0}(t)\right)$ are less than $\delta_{1}$ except on sets of measure less than or equal to $\delta_{1}$.

Both quantities are less than $\delta_{1}$ on a set $A$ of measure no less than $1-2 \delta_{1}$, so by definition of $\delta_{1}, \nu\left(f g^{-1}(t), f_{0} g_{0}^{-1}(t)\right)<\varepsilon / 3$ on $A$, and $\int_{A} \nu\left(f g^{-1}(t), f_{0} g_{0}^{-1}(t)\right) d t<$ $\varepsilon / 3$. Moreover, writing $B=[0,1) \backslash A$ and using (1), we have

$$
\begin{aligned}
\int_{B} \nu\left(f g^{-1}(t), f_{0} g_{0}^{-1}(t)\right) d t \leqslant & \int_{B} \nu\left(f(t), f_{0}(t)\right) d t \\
& +\int_{B} \nu\left(g(t), g_{0}(t)\right) d t+2 \int_{B} \nu\left(g_{0}(t), e\right) d t \\
\leqslant & \nu^{*}\left(f, f_{0}\right)+\nu^{*}\left(g, g_{0}\right)+4 M \delta_{1} \\
< & 2 \delta+\varepsilon / 3<2 \varepsilon / 3,
\end{aligned}
$$

and so $\nu^{*}\left(f g^{-1}, f_{0} g_{0}^{-1}\right)=\int_{0}^{1} \nu\left(f g^{-1}(t), f_{0} g_{0}^{-1}(t)\right) d t<\varepsilon$, and the function $(f, g) \mapsto$ $\mathrm{fg}^{-1}$ is continuous.

The path-connectedness of $G^{*}$ can be proved directly by adapting the proof of Theorem 1 of [2]. However we shall give a proof which uses facts already known about the Brown-Morris topology on $G^{*}$. We denote the latter topology by $\tau$.

For each integer $k \geqslant 1$, the (left-invariant) pseudometric $\nu_{k}$ defined by $\nu_{k}(x, y)$ $=\min (k, \nu(x, y))$ is equivalent to $\nu$, and since it is also bounded, Proposition 5 of [2] shows that the extension $\nu_{k}^{*}$ induces the topology $\tau$ on $G^{*}$. Set $G_{n}^{*}=\left\{f \in G^{*}\right.$ : $\nu(f(t), e) \leqslant n$ for all $t \in[0,1)\}$ for each integer $n \geqslant 1$. Theorem 1 of [2] shows that $\left(G^{*}, \tau\right)$ is path-connected, and the proof of that theorem shows clearly that two points in $G_{n}^{*}$ are in fact connected by a path lying wholly in $G_{n}^{*}$, so that $\left(G_{n}^{*}, \tau\right)$ is path-connected for each $n$. But it is easy to see that $\nu^{*}$ and $\nu_{2 n}^{*}$ are equal on $G_{n}^{*}$, and so $\left(G_{n}^{*}, \tau(\nu)\right)$ is path-connected. Then since $G^{*}$ is the union of the increasing sequence of path-connected subsets $\left\{G_{n}^{*}\right\}$, we see that $\left(G^{*}, \tau(\nu)\right)$ is path-connected as required.

THEOREM 2. Let $X$ be a completely regular Hausdorff space and $F(X)$ the (Markov) free topological group on $X$. If $H$ is the subgroup of $F(X)$ generated by a finite subset $\left\{x_{1}, x_{2}, \ldots, x_{n}\right\}$ of $X$, then any norm $N$ on $H$ extends to a continuous norm on $F(X)$.

Proof. We remark that $H$ is the free group on $\left\{x_{1}, \ldots, x_{n}\right\}$, and has the discrete topology, by Theorem 1.11 of [4], so that all its norms are continuous. For $h \in H$, set $L(h)$ equal to the reduced word length of $h$ with respect to $X$; it is immediate that $L$ is a continuous norm on $H$. The corresponding left-invariant metric $\lambda$ induces the discrete topology on $H$ (since all nonzero distances are at least one) and so, by Theorem $1, H^{*}$ with the topology $\tau(\lambda)$ induced by the extended metric $\lambda^{*}$ is a path-connected topological group.

By virtue of this and the fact that $X$ is completely regular, there is a continuous function $\phi: X \rightarrow H^{*}$ with the property that $\phi\left(x_{i}\right)(t)=x_{i}, t \in[0,1)$, for each $i$. Since $H^{*}$ is a topological group we may extend $\phi$ to a continuous homomorphism $\Phi: F(X) \rightarrow H^{*}$. Let $N^{*}$ be the extension of $N$ to $H^{*}$. We claim that the norm $\bar{N}=N^{*} \Phi$ on $F(X)$ is the continuous extension of $N$ that we require. 
To see this, note firstly that since $\phi\left(x_{i}\right)(t)$ is identically $x_{i}$ for each $i, \Phi(h)(t)$ is identically $h$ for each $h \in H$, so that $\bar{N}(h)=N^{*} \Phi(h)=\int_{0}^{1} N(\Phi(h)(t)) d t=N(h)$.

Secondly, setting $M=\max \left\{N\left(x_{1}\right), \ldots, N\left(x_{n}\right)\right\}$, we see that $N(h)<M L(h)$ for $h \in H$, and hence that $N^{*}(f) \leqslant M L^{*}(f)$ for $f \in H^{*}$. Then if $\varepsilon>0$, the set $U=\left\{f \in H^{*}: L^{*}(f)<\varepsilon / M\right\}$ is an open neighbourhood of the identity in $\left(H^{*}, \tau(\lambda)\right.$ ) such that $N^{*}(U) \subseteq[0, \varepsilon)$ (assuming $M \neq 0$, as we clearly may). This shows that $N^{*}$ is continuous at the identity, and it follows from the definition of a norm that $N^{*}$ is continuous everywhere. Hence the composite $\bar{N}=N^{*} \Phi$ is also continuous, completing the proof.

REMARK. At least in the case when $N=L$, the extension $N^{*}$ is not continuous on $H^{*}$ when $H^{*}$ is given the usual Brown-Morris topology; this is easily seen by adapting the example given in [1].

\section{REFERENCES}

1. Kevin Bicknell and Sidney A. Morris, Norms on free topological groups, Bull. London Math. Soc. 10 (1978), 280-284.

2. Ronald Brown and Sidney A. Morris, Embeddings in contractible or compact objects, Colloq. Math. 38 (1978), 213-222.

3. S. Hartman and J. Mycielski, On the imbedding of topological groups into connected topological groups, Colloq. Math. 5 (1958), 167-169.

4. Sidney A. Morris, Varieties of topological groups. II, Bull. Austral. Math. Soc. 2 (1970), 1-13.

Department of Pure Mathematics, la Trobe University, Bundoora, Victoria, Australa 3083

Department of Mathematics, University of QueEnsland, St. Lucia QueEnsland, Australia 4067 